\title{
Manual Joints Staggered Joints Therapy for Acute Chest Pain: A Case Report
}

\author{
Gang Luo ${ }^{1}$, Weipeng Rong ${ }^{1}$, Lixia Li $^{2}$
}

\author{
${ }^{1}$ Department of Traditional Chinese Medicine Rehabilitation, HaiZhuQuJiangHaiJie Community Health Service \\ Centre, Guangzhou, 510305, China \\ ${ }^{2}$ Department of Acupuncture, Guangzhou Hospital of Traditional Chinese Medicine, Guangzhou, 510130, China
}

\begin{abstract}
Background: Chest pain is a common clinical condition and the reasons for it are complex. Case Report: One categorization system describes nine types, one of which includes bone diseases such as pathological changes in the cervical vertebrae or thoracic vertebrae. Herein we describe the case of a 45-year-old woman with chest pain that was successfully cured after three joints staggered joints therapy.
\end{abstract}

Keywords: Chest Pain, Joints Staggered Joints, Manual Therapy

\begin{abstract}
Introduction
"Chest pain" is a broad term used to refer to pain localized to the chest, some of which may radiate into the waist, back, and abdomen within the lumbar spinal region associated with the vertebral column or surrounding musculature ${ }^{1}$. Jameson has described nine categories of chest pain, including that associated with bone conditions such as pathological changes in the cervical vertebra or thoracic vertebra ${ }^{2}$. The most common reason is joint disorder due to long-term adoption of unsuitable posture ${ }^{3}$. Regardless of the cause, joint disorders in any workers are a concern with regard to health and wellbeing, career progression, and associated medical costs $\mathrm{s}^{4,5}$. In a previously reported osteopathic trial there was a substantial reduction in high baseline pain severity score and an increase in back-specific function in patients treated with manual therapy ${ }^{6}$. The use of joints staggered joints to treat chest pain caused by joint disorders is a novel concept.
\end{abstract}

\section{Case report}

A 45-year-old woman was admitted to a major hospital on 10 August 2019 reporting that she had been suffering from chest pain and back pain for the last 5 days. She stated that she thought her pain was caused by working until late into the night. Examinations were conducted including color Doppler echocardiography of the heart, electrocardiography, chest spiral computed tomography, and cardiac function, but the results were all normal. There was initially no definitive diagnosis and she was prescribed nitroglycerin, musk heart protecting pills. The pills evidently only provided minimal and temporary relief however, so she returned to our hospital for further diagnosis and treatment.

On the second visit the patient exhibited clear consciousness but complained of persistent tiredness, chest pain, left back pain, palpitations, and poor sleep. Her appetite was reportedly normal and there was no nausea or vomiting. Physical examination revealed that the third and fourth thoracic vertebrae were discontinuous and not level, and the T3-T4 spinal processes were markedly tender. The symptoms were exacerbated during lateral bending. She was diagnosed with disturbance of small joints of the thoracic vertebrae. Risks of manual therapy were discussed with the patient, and consent was obtained for its administration.

Patients were instructed to relax before surgery, manual functional repositioning of the small joints of the thoracic vertebrae was conducted as follows. After placing the patient in a sitting position with her hands crossed behind her head, an operator stood behind her and held her elbows, with one of his knees against the exacerbated cervical spine injury. Operatives pulled the elbows back until the required range was achieved. He then pulled back once again, over a small range, forcibly and quickly. Lastly, the operatives quickly release the patient's hand. There was audible joint clicking during the therapy, suggesting that it had been successful.

During such procedures care must be taken to ensure that the patient is relaxed and not resistant. Actions should be performed accurately, and firmly but not violently during the therapy. The patient should be advised to engage in active muscle exercise after the procedures.

In the current patient the pain and associated symptoms improved substantially after the first treatment, and she was healed after the third treatment. She was instructed to continue stretching techniques at home and to attend the clinic for routine follow-up, and she reported that there was no recurrence of symptoms at a 3-month follow-up. The patient has provided written informed consent for the publication of this report. 


\section{Discussion}

Chest pain is a common medical complaint. Even when cardiac factors are involved it is easy for it to be misdiagnosed as a non-cardiogenic condition because of symptom similarities, however, we must cervical rotatory manipulation of thoracic vertebrae from internal diseases ${ }^{7}$. This requires professional competence and a broad knowledge base, in order to formulate precise diagnoses and differentiate between varieties of disease accurately. Despite probable underreporting, there are many informative reports and studies that provide insight into the problem. Treatment plans that can improve the patient's quality of life and reduce their healthcare expenses are optimal. Joints staggered joints is an easy and safe choice for primary doctors.

The authors declare no competing interests.

\section{References}

1. Bösner S, Becker A, Hani MA, Keller H, Sönnichsen AC, Karatolios $\mathrm{K}$, et al. Chest wall syndrome in primary care patients with chest pain: presentation, associated features and diagnosis. Family practice. 2010;27(4):363-9.

2. Jameson J. Harrison's Endocrinology, 3E: McGraw-Hill Education; 2013.

3. Wikström B-O, Kjellberg A, Landström U. Health effects of long-term occupational exposure to whole-body vibration: a review. International journal of industrial ergonomics. 1994;14(4):273-92.

4. Liem R, Rayman P. Health and social costs of unemployment: Research and policy considerations. American Psychologist. 1982;37(10):1116.

5. Yang F, Yang L, Ren E, Yang Y, Wang J, Kang X. Surgical Treatment of Ankylosing Spondylitis with Andersson Lesion. Journal of the College of Physicians and Surgeons Pakistan. 2019;29(2):S135-S7.

6. Andicochea CT, Fulkerson J, Taylor BM, Portouw SJ. Manual therapy for chronic low back pain in an F-5 pilot. Military medicine. 2015;180(10):e1132-e5.

7. Kar-mun CW, Schneider JI. High-risk chief complaints I: chest pain-the big three. Emergency Medicine Clinics. 2009;27(4):685-712. 